\title{
Biomechanical comparison of bioresorbable F-u-HA/PLLA screw and tension band wire fixation in medial malleolar fractures
}

\author{
HIROAKI SAKAI $^{1}$, NAOYA TAKADA ${ }^{2}$, GEN KUROYANAGI ${ }^{1,3}$, \\ TAKUYA USAMI $^{1}$, YOSHINO UEKI ${ }^{3}$ and HIDEKI MURAKAMI ${ }^{1}$ \\ ${ }^{1}$ Department of Orthopedic Surgery, Nagoya City University Graduate School of Medical Sciences, Nagoya, Aichi 467-8601; \\ ${ }^{2}$ Department of Orthopedic Surgery, Kainan Hospital, Yatomi, Aichi 498-8502; ${ }^{3}$ Department of Rehabilitation Medicine, \\ Nagoya City University Graduate School of Medical Sciences, Nagoya, Aichi 467-8601, Japan
}

Received December 18, 2020; Accepted March 26, 2021

DOI: $10.3892 /$ wasj.2021.97

\begin{abstract}
The authors have recently reported that forged composites of raw particulate unsintered hydroxyapatite/poly-L-lactide (F-u-HA/PLLA) screws are useful for the treatment of medial malleolar fracture. However, the biomechanical properties of these screws have not yet been fully elucidated. The aim of the present study was to compare the biomechanical properties of F-u-HA/PLLA screws with those of tension band wiring (TBW) using medial malleolar fracture models obliquely oriented at $45^{\circ}$ angles in synthetic tibial bones. Fractures were fixed using two 4.5-mm-diameter partial thread F-u-HA/PLLA screws, two 4.5-mm-diameter full thread F-u-HA/PLLA screws, or TBW. The fracture models were tested parallel to the distal joint surface and parallel to the fracture line. The compression load at 1, 2, 3, 4 and $5 \mathrm{~mm}$ of fracture displacement was assessed. The compression load parallel to the distal joint surface at 3,4 and $5 \mathrm{~mm}$ of fracture displacement was significantly higher in the partial thread screw group than in the TBW group $(\mathrm{P}<0.01)$. Additionally, the compression load parallel to the distal joint surface at 4 and $5 \mathrm{~mm}$ was significantly higher in the full thread screw group than in the TBW group $(\mathrm{P}<0.001)$. However, the TBW group exhibited a higher compression load at $1 \mathrm{~mm}$ compared to the full thread group $(\mathrm{P}<0.05)$. As regards the compression load parallel to the fracture line, the compression load at 3,4 and $5 \mathrm{~mm}$ of fracture displacement was markedly higher in the partial thread screw group than in the TBW group $(\mathrm{P}<0.01)$; likewise, the compression load at 4 and $5 \mathrm{~mm}$ of displacement was significantly higher in the full thread screw group than in the TBW group $(\mathrm{P}<0.001)$. On the whole, these results strongly
\end{abstract}

Correspondence to: Dr Gen Kuroyanagi, Department of Orthopedic Surgery, Nagoya City University Graduate School of Medical Sciences, 1 Kawasumi, Mizuho-cho, Mizuho-ku, Nagoya, Aichi 467-8601, Japan

E-mail: kokuryugen@yahoo.co.jp

Key words: F-u-HA/PLLA screw, bioresorbable screw, tension band wiring, medial malleolar fracture, biomechanical property suggest that F-u-HA/PLLA screw fixation, particularly partial thread screw fixation, has good biomechanical strength and provides adequate stability for the treatment of medial malleolar fractures.

\section{Introduction}

Medial malleolar fractures are very common and are associated with ankle injury (1). Since the medial malleolus plays a pivotal role in the stability and function of the ankle joint, anatomical reduction by surgery is generally recommended for displaced medial malleolar fractures (2). Reportedly, open reduction and internal fixation restores articular congruency and prevents post-traumatic arthritis (2). A variety of techniques for medial malleolar fracture repair employ metallic implants, including metallic screws and tension band wiring (TBW) by Kirshner wires (K-wires) and stainless-steel wire (3-5). Although a number of studies have described good clinical outcomes following surgical treatment, post-operative complications, such as residual pain, skin irritation and the backing out of implants caused by metallic implants are problematic (3-5). Moreover, in some cases, the patient requires the removal of the metallic implant due to these post-operative complications (6).

The implant bioactivity, bioabsorbability and high mechanical strength of forged composites of raw particulate unsintered hydroxyapatite/poly-L-lactide (F-u-HA/PLLA) are similar to those of human cortical bone (7). The authors have previously demonstrated the utility of F-u-HA/PLLA screws and pins for the treatment of tibial plateau fractures and distal humeral fractures $(8,9)$. Furthermore, F-u-HA/PLLA screws have been proven effective for the treatment of medial malleolar fractures, demonstrating good clinical results without any post-operative complications, such as deep infections, late aseptic tissue response, or foreign body reactions (10). Additionally, none of the patients required the removal of these screws (10). However, to the best of our knowledge, there are no reports available to date of mechanical comparisons between F-u-HA/PLLA screws and TBW in medial malleolar fractures. Thus, the aim of the present study was to compare the biomechanical properties of partial or full thread F-u-HA/PLLA screws with TBW in a medial malleolar fracture model using Synbone ${ }^{\circledR}$ AG. 
A

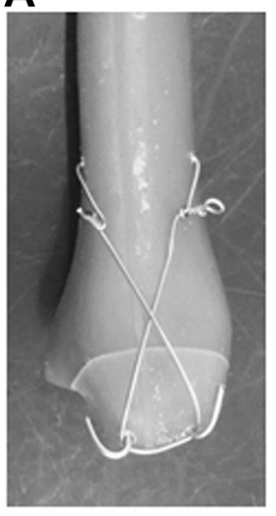

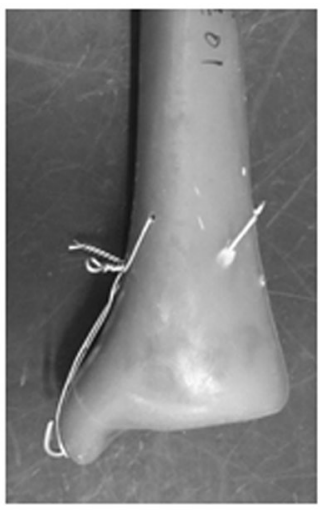

B

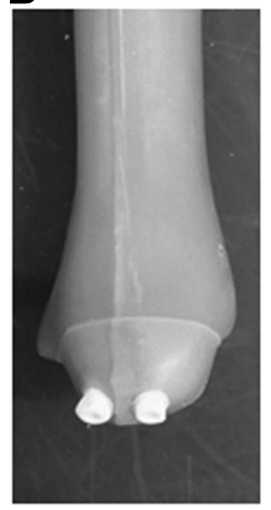

C

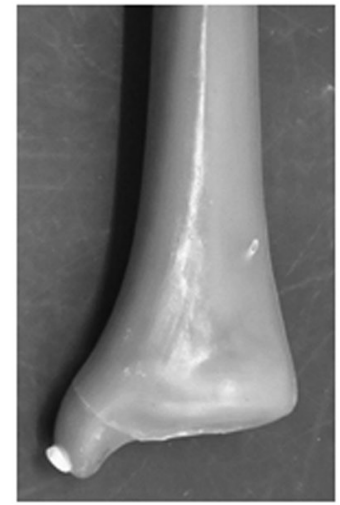

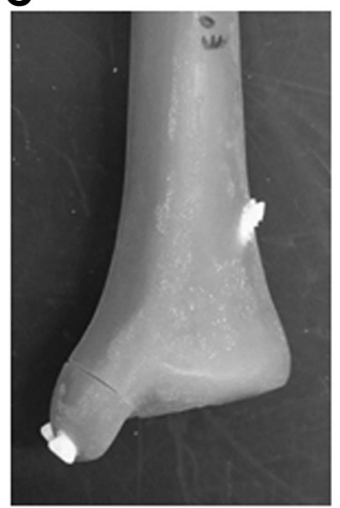

Figure 1. (A) The tension band wiring group was fixed using two 1.6-mm-diameter K-wires and a 0.9-mm-diameter metallic soft wire. (B) The partial thread screw group was fixated with two partial thread $4.5 \times 45 \mathrm{~mm}$ unsintered hydroxyapatite particles/poly-L-lactide (F-u-HA/PLLA) screws. (C) The full thread screw group was fixated with two full thread 4.5x70 mm F-u-HA/PLLA screws.

\section{A}

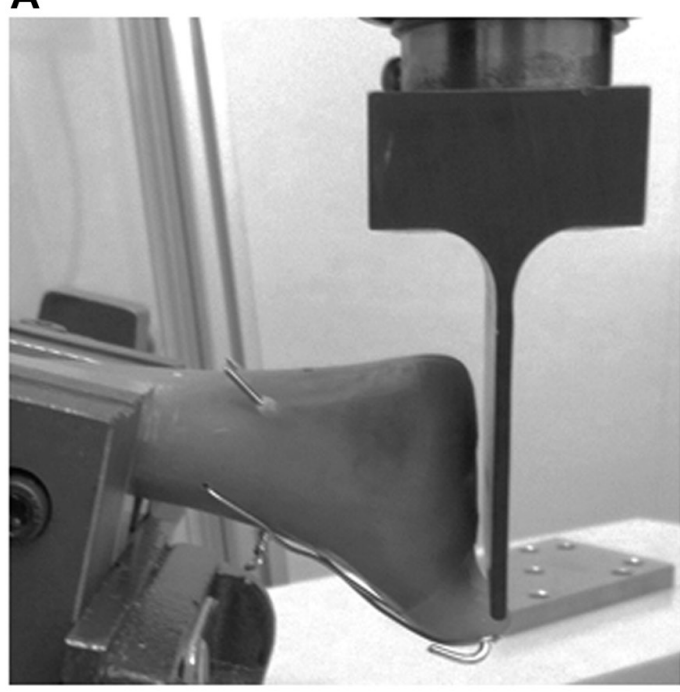

B

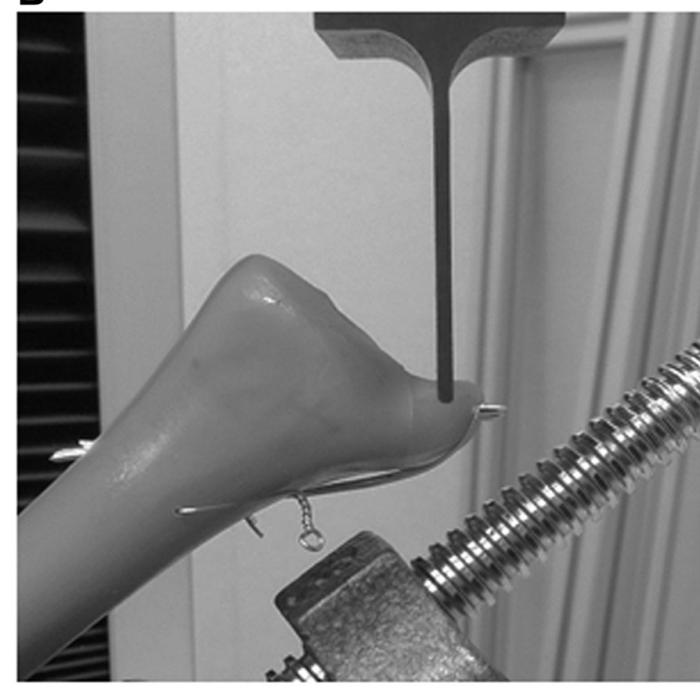

Figure 2. Image showing the tension loading mechanism. (A) Compression load parallel to the distal joint surface (B) Compression load parallel to the fracture line.

\section{Materials and methods}

Fracture models and grouping. A total of 42 fourth generation composite synthetic left tibial bones (Synbone ${ }^{\circledR}$ AG) were used in the present study. The tibial bone was cut $17 \mathrm{~cm}$ from the distal articular surface. Medial malleolar fractures obliquely oriented at $45^{\circ}$ angles to the longitudinal axis of the tibial bone were created using a saw.

The 42 fracture models were divided into three groups as follows: a TBW group, a partial thread screw group and a full thread screw group ( $n=14$ per group). The TBW group $(n=14)$ underwent traditional TBW using two 1.6-mm-diameter $\mathrm{K}$-wires and a 0.9 -mm-diameter stainless-steel wire (Mizuho Co., Ltd.) (Fig. 1A). The stainless-steel wire was passed through the tibia on the proximal side of the fracture line, and then tightened in a figure-eight pattern. The tension of the stainless-steel wire was kept constant at $30 \mathrm{~N}$ by a scale device. The partial thread screw group $(n=14)$ was fixed with two partial thread $4.5 \times 45 \mathrm{~mm}$ F-u-HA/PLLA screws
(Osteotrans Puls ${ }^{\circledR}$, Teijin Medical Technologies Co., Ltd.), which were fixed mono-cortically (Fig. 1B). On the other hand, the full thread screw group $(n=14)$ was fixed with two full thread $4.5 \times 70 \mathrm{~mm}$ F-u-HA/PLLA screws that were fixed bi-cortically (Fig. 1C). The universal testing machine (AG-20kNXD, Shimadzu Corporation) tested the tibial bone models under two compression loading conditions. The load was applied parallel to the distal tibial joint surface and parallel to the fracture line at $10 \mathrm{~mm} / \mathrm{min}$, respectively (Fig. 2). The direction of the load was simulated as the force received from the talus during walking and the force pushed up by the talus during a fracture. Compression loads at 1,2,3,4 and $5 \mathrm{~mm}$ of fracture displacement were analyzed.

Statistical analysis. Statistical analyses were performed with SPSS version 23 statistical software (IBM Co.). Data were analyzed using one-way analysis of variance (ANOVA) with Tukey's honestly significant difference (HSD) post hoc test for multiple comparisons. A P-value $<0.05$ was 


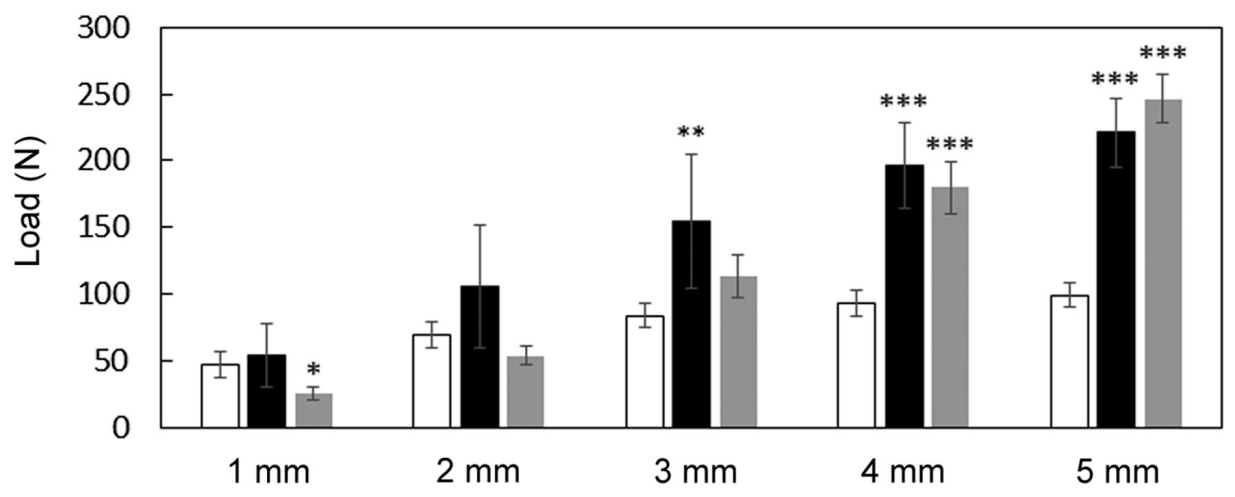

पTBW $\square$ Partial thread $\square$ Full thread

Figure 3. Compression load parallel to the distal joint surface was calculated under the fracture displacement. Each value represents the mean \pm standard error of the mean (SEM) of seven determinations from independent model preparations. ${ }^{*} \mathrm{P}<0.01,{ }^{* * *} \mathrm{P}<0.001$ and ${ }^{* * * *} \mathrm{P}<0.001$ compared to the value of the TBW group. TBW, tension band wiring.

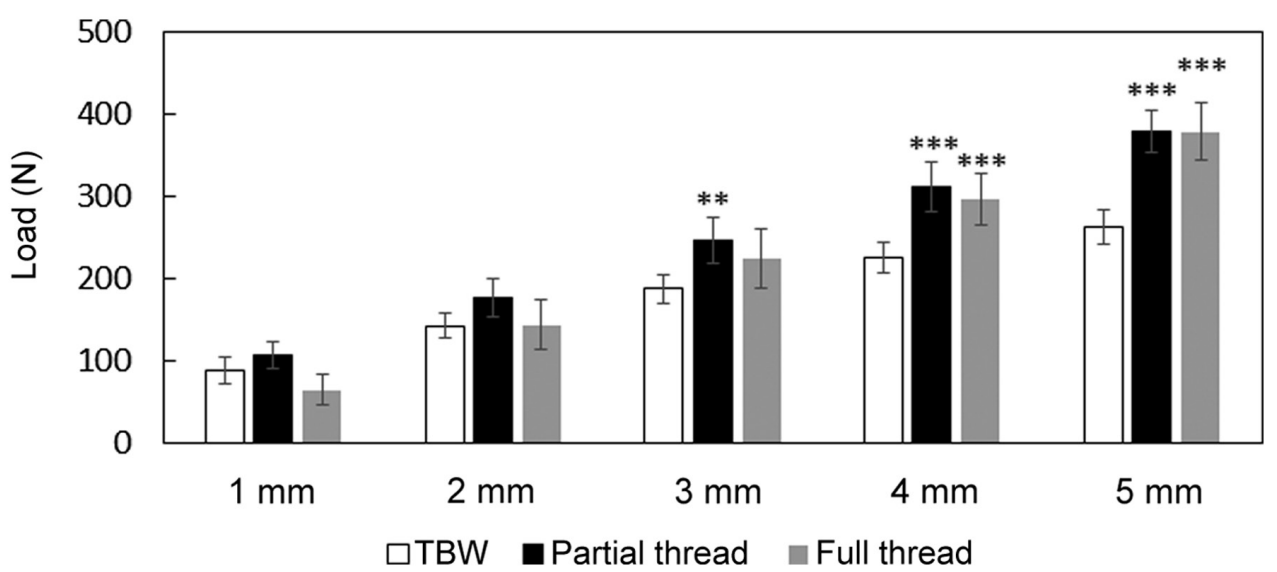

Figure 4. Compression load parallel to the fracture line was calculated under the fracture displacement. Each value represents the mean \pm standard error of the mean (SEM) of seven determinations from independent model preparations. ${ }^{* *} \mathrm{P}<0.01$ and ${ }^{* * *} \mathrm{P}<0.001$ compared to the value of the TBW group. TBW, tension band wiring.

considered to indicate a statistically significant difference. All data are presented as the mean \pm standard error of the mean (SEM) of seven determinations from independent model preparations.

\section{Results}

The compression load parallel to the distal joint surface at 3,4 and $5 \mathrm{~mm}$ of fracture displacement in the partial thread screw group was significantly higher than that in the TBW group (3 mm: TBW, $84 \pm 8 \mathrm{~N}$; partial thread, $154 \pm 50 \mathrm{~N}$; $4 \mathrm{~mm}$ : TBW, $93 \pm 9 \mathrm{~N}$; partial thread, $196 \pm 32 \mathrm{~N} ; 5 \mathrm{~mm}$ : TBW, $99 \pm 10 \mathrm{~N}$; partial thread, $221 \pm 26 \mathrm{~N}$; P<0.01). Similar to the partial thread screw, the compression load parallel to the distal joint surface at 4 and $5 \mathrm{~mm}$ of fracture displacement in the full thread screw group was significantly higher than that in the TBW group (full thread: $4 \mathrm{~mm}, 180 \pm 19 \mathrm{~N} ; 5 \mathrm{~mm}$, $246 \pm 19 \mathrm{~N} ; \mathrm{P}<0.001)$. However, the compression load parallel to the distal joint surface at $1 \mathrm{~mm}$ of fracture displacement in the full thread screw group was significantly lower than that in the TBW group ( $1 \mathrm{~mm}$ : TBW, $47 \pm 6 \mathrm{~N}$; full thread, $25 \pm 5 \mathrm{~N}$; $\mathrm{P}<0.05$ ) (Fig. 3).
As regards the compression load parallel to the fracture line, the partial thread screw group exhibited a significantly higher compression load than the TBW group between 3 and $5 \mathrm{~mm}$ (3 mm: TBW, $187 \pm 18 \mathrm{~N}$; partial thread, $245 \pm 28 \mathrm{~N}$; $4 \mathrm{~mm}$ : TBW, $225 \pm 18 \mathrm{~N}$; partial thread, $310 \pm 30 \mathrm{~N} ; 5 \mathrm{~mm}$ : TBW, $262 \pm 20 \mathrm{~N}$; partial thread, $378 \pm 25 \mathrm{~N}$; P<0.01). The compression load parallel to the fracture line at 4 and $5 \mathrm{~mm}$ of fracture displacement in the full thread screw group was significantly higher than that in the TBW group (full thread: $4 \mathrm{~mm}, 295 \pm 32 \mathrm{~N} ; 5 \mathrm{~mm}, 378 \pm 34 \mathrm{~N} ; \mathrm{P}<0.001)$. There were no significant differences in the compression load parallel to the fracture line at 1 and $2 \mathrm{~mm}$ of fracture displacement between the TBW group and the partial or full thread screw groups (Fig. 4). No breakage of the F-u-HA/PLLA screws or TBW was observed when the gap between fractures reached $5 \mathrm{~mm}$.

\section{Discussion}

The present study demonstrated that partial thread F-u-HA/PLLA screws are stronger than TBW in terms of the fixation of medial malleolar fractures. On the other hand, full thread F-u-HA/PLLA screws also exhibited stronger 
Table I. Material properties of the u-HA/PLLA composite.

\begin{tabular}{lrl}
\hline Property & \multicolumn{1}{c}{ Value } & Unit \\
\hline Viscosity average molecular weight & \multicolumn{1}{c}{210} & $\mathrm{kDa}$ \\
Bending strength & $269.2( \pm 2.5)$ & $\mathrm{MPa}$ \\
Bending modulus & $7.6( \pm 0.1)$ & $\mathrm{GPa}$ \\
Tensile strength & $121.5( \pm 6.2)$ & $\mathrm{MPa}$ \\
Tensile modulus & $2.3( \pm 0.2)$ & $\mathrm{GPa}$ \\
Compressive strength & $106.7( \pm 3.7)$ & $\mathrm{MPa}$ \\
Shear strength & $126.4( \pm 3.3)$ & $\mathrm{MPa}$ \\
Torsional strength $(\varnothing 3.2 \mathrm{~mm})$ & $64.7( \pm 1.0)$ & $\mathrm{N} \cdot \mathrm{cm}$ \\
\hline
\end{tabular}

compression loads with a displacement of $>4 \mathrm{~mm}$ under both conditions, parallel to the distal joint surface and parallel to the fracture line, compared to TBW. However, when the displacement was $1 \mathrm{~mm}$ parallel to the distal joint surface, the compression load tolerance of full thread F-u-HA/PLLA screws was lower than that of TBW. TBW is usually firmly fixed by crimping even when the displacement of the fractured part is small. On the other hand, following a displacement of $2 \mathrm{~mm}$, since the tension of the stainless steel wire did not function and the K-wire itself was easily pulled out, it was probable that the value of the compression load remained low. As for the full thread screws, as the full thread screw is bi-cortically fixed and not backed out, it is likely that the tensile strength of the screw greatly affected the load value.

It has been reported that full thread metal screws have a higher strength than that of partial thread metal screws for the fixation of medial malleolar fractures $(3,11)$. Fowler et al (11) reported that bi-cortical screw fixation by full thread screws is superior to uni-cortical screw fixation by partial thread screws in terms of transverse (vs. axial) compression loads at $2 \mathrm{~mm}$ of displacement using tibial sawbone fracture models. Downey et al (12) also reported that full thread screws exhibited a higher initial construct stiffness and catastrophic failure strength compared to partial thread screws following shear axial loading using sawbone blocks. Thus, when using the metal implant, it is likely that bi-cortical screw fixation by full thread screws has an advantage over partial thread screws. However, the present study demonstrated that the compression load of the partial thread F-u-HA/PLLA screw group was stronger in displacements both parallel to the distal joint surface and parallel to the fracture line than that of the full thread F-u-HA/PLLA screw group. Based on these findings, the mechanical properties of F-u-HA/PLLA screws and metallic screws differ in medial malleolar fractures. Thus, further studies on screw suitability in the treatment of medial malleolar fractures are required. The screws used in the present study are made from composites of uncalcined and unsintered HA particles and PLLA. The material properties of u-HA/PLLA composite are summarized in Table I.

TBW fixation is one of the most common treatments recommended by the Arbeitsgemeinschaft für Osteosynthesefragen (AO) group for medial malleolar fractures (13). It has been shown that fixation by TBW is stronger than fixation by uni-cortical partial thread metallic screws using a cadaver model (5). However, due to post-operative complications caused by metallic wire and pins, implant removal is often required (6). Recently, the AO group recommended fixation using two parallel partial threads cancellous screws for the treatment of medial malleolar fractures (14). Previously, the authors demonstrated that F-u-HA/PLLA partial thread screws were effective for the treatment of medial malleolar fractures without any post-operative complications (10). Additionally, the results of the present study demonstrated that F-u-HA/PLLA partial thread screws were stronger than TBW. Taking these findings into account, F-u-HA/PLLA partial thread screws may be an alternative option for the treatment of medial malleolar fractures.

F-u-HA/PLLA implants have several advantages, including bioactivity, bioresorbability and a high mechanical strength (7). These implants also have good biocompatibility and osteoconductivity, which stimulate direct bone-bonding and new bone formation $(15,16)$. Thus, F-u-HA/PLLA implants are currently utilized in several clinical fields, such as orthopedic, maxillofacial and trauma surgeries (17-19). Compared to other bioresorbable PLLA implants, the F-u-HA/PLLA implant has higher initial mechanical strength than human cortical bone, which has a strength of $200 \mathrm{MPa}$ (7). Shikinami and Okuno (7) reported that the F-u-HA/PLLA material had an initial bending strength of $270 \mathrm{MPa}$, which is much higher than that of natural cortical bone. In a previous study by the authors, the breakage of F-u-HA/PLLA screws was not observed following the treatment of medial malleolar fractures (10). Although there is no doubt that F-u-HA/PLLA screws are generally not applicable in the treatment of load-bearing joints, F-u-HA/PLLA screws seem to be strong enough for use in this area.

There are several limitations to the present study. First, Synbone ${ }^{\circledR}$ AG was used in the present study. Synbone ${ }^{\circledR}$ AG is a synthetic sawbone and is known to have biomechanical properties similar to those of human bone (20). However, Synbone ${ }^{\circledR}$ AG mimics normal healthy bone structure; thus, the present results may not be applicable to patients with osteoporosis. Second, the mechanical test was performed under only two load conditions. In a clinical setting, the fracture site is subject to a variety of forces from various directions through the surrounding tissues. Thus, further research with other load conditions will be required to elucidate the detailed mechanical properties of the F-u-HA/PLLA screws. Finally, medial malleolus fracture with fracture line located at $45^{\circ}$ to the joint space is not the most common in clinical practice. Generally, medial malleolus fractures can be distinguished from fracture types, such as parallel or perpendicular location to the joint line. Forces acting on different types of malleolar fractures may have different influence on the screws. Therefore, it seems likely that the results presented herein may not be directly applicable to clinical practice. Further investigations are required to clarify the effects of different fracture patterns on the screws.

In conclusion, the present study demonstrates that F-u-HA/PLLA screw fixation has a good biomechanical strength and provides adequate stability for the treatment of medial malleolar fractures. The F-u-HA/PLLA screw may prove to be a useful option for the treatment of medial malleolar fractures. 


\section{Acknowledgements}

The F-u-HA/PLLA screws, Synbone ${ }^{\circledR}$ AG, and the universal testing machine were kindly provided by Takiron Co. Ltd.

\section{Funding}

The present study was supported in part by a Grant-in-Aid for Scientific Research (19K18471) from the Ministry of Education, Culture, Sports, Science and Technology of Japan.

\section{Availability of data and materials}

The datasets used and/or analyzed during the current study are available from the corresponding author on reasonable request.

\section{Authors' contributions}

HS and NT, YU and HM designed the study. HS, NT and TU performed the experiments. HS, NT and GK analyzed the data. HS, NT and GK drafted the manuscript. NT, YU and HM critically reviewed the manuscript. HS and GK confirm the authenticity of all the raw data. All authors have read and approved the final manuscript.

\section{Ethics approval and consent to participate}

No human tissues were used in the present study; only synthetic bone tissue was used.

\section{Patient consent for publication}

Not applicable.

\section{Competing interests}

The authors declare that they have no competing interests.

\section{References}

1. Lokerman RD, Smeeing DPJ, Hietbrink F, van Heijl M and Houwert RM: Treatment of a Scientifically Neglected Ankle Injury: The Isolated Medial Malleolar Fracture. A Systematic Review. J Foot Ankle Surg 58: 959-968, 2019.

2. Carter TH, Duckworth AD and White TO: Medial malleolar fractures: Current treatment concepts. Bone Joint J 101B: 512-521, 2019.

3. Yammine K, Karam K and Assi C: Cortical versus cancellous screws in treating medial malleolar fractures: A systematic review of comparative clinical and biomechanical studies. Foot 40: 81-86, 2019.

4. Barnes H, Cannada LK and Watson JT: A clinical evaluation of alternative fixation techniques for medial malleolus fractures. Injury 45: 1365-1367, 2014

5. Ebraheim NA, Ludwig T, Weston JT, Carroll T and Liu J: Comparison of surgical techniques of 111 medial malleolar fractures classified by fracture geometry. Foot Ankle Int 35: 471-477, 2014
6. Brown OL, Dirschl DR and Obremskey WT: Incidence of hardware-related pain and its effect on functional outcomes after open reduction and internal fixation of ankle fractures. J Orthop Trauma 15: 271-274, 2001.

7. Shikinami Y and Okuno M: Bioresorbable devices made of forged composites of hydroxyapatite (HA) particles and poly-L-lactide (PLLA): Part I. Basic characteristics. Biomaterials 20: 859-877, 1999.

8. Kuroyanagi G, Yoshihara H, Yamamoto N, Suzuki H, Yamada K, Yoshida Y, Otsuka T and Takada N: Treatment of Lateral Tibial Condylar Fractures Using Bioactive, Bioresorbable Forged Composites of Raw Particulate Unsintered Hydroxyapatite/Poly-L-Lactide Screws. Orthopedics 41: e365-e368, 2018

9. Takada N, Suzuki H, Yamada K and Otsuka T: Treatment of Ankle Fractures Using New High-Strength, Bioactive, Bioresorbable Forged Composites of Unsintered Hydroxyapatite/poly-L-lactide (F-u-HA/PLLA) Implants. J Orthop 7: e5, 2010.

10. Takada N, Otsuka T, Suzuki H and Yamada K: Pediatric displaced fractures of the lateral condyle of the humerus treated using high strength, bioactive, bioresorbable F-u-HA/PLLA pins: A case report of 8 patients with at least 3 years of follow-up. J Orthop Trauma 27: 281-284, 2013.

11. Fowler TT, Pugh KJ, Litsky AS, Taylor BC and French BG: Medial malleolar fractures: A biomechanical study of fixation techniques. Orthopedics 34: e349-e355, 2011.

12. Downey MW, Kosmopoulos V and Carpenter BB: Fully Threaded Versus Partially Threaded Screws: Determining Shear in Cancellous Bone Fixation. J Foot Ankle Surg 54: 1021-1024, 2015.

13. Meinberg EG, Agel J, Roberts CS, Karam MD and Kellam JF: Fracture and dislocation classification compendium-2018. J Orthop Trauma 32 (Suppl 1): S1-S170, 2018.

14. Parker L, Garlick N, McCarthy I, Grechenig S, Grechenig W and Smitham P: Screw fixation of medial malleolar fractures: A cadaveric biomechanical study challenging the current $\mathrm{AO}$ philosophy. Bone Joint J 95-B: 1662-1666, 2013.

15. Furukawa T, Matsusue Y, Yasunaga T, Nakagawa Y, Okada Y, Shikinami Y, Okuno M and Nakamura T: Histomorphometric study on high-strength hydroxyapatite/poly(L-lactide) composite rods for internal fixation of bone fractures. J Biomed Mater Res 50: 410-419, 2000.

16. Hasegawa S, Ishii S, Tamura J, Furukawa T, Neo M, Matsusue Y, Shikinami Y, Okuno M and Nakamura T: A 5-7 year in vivo study of high-strength hydroxyapatite/poly(L-lactide) composite rods for the internal fixation of bone fractures. Biomaterials 27: 1327-1332, 2006.

17. Lind M, Feller J and Webster KE: Tibial bone tunnel widening is reduced by polylactate/hydroxyapatite interference screws compared to metal screws after ACL reconstruction with hamstring grafts. Knee 16: 447-451, 2009.

18. Li ZH, Yu AX, Guo XP, Qi BW, Zhou M and Wang WY: Absorbable implants versus metal implants for the treatment of ankle fractures: A meta-analysis. Exp Ther Med 5: 1531-1537, 2013.

19. Landes C, Ballon A, Ghanaati S, Tran A and Sader R: Treatment of malar and midfacial fractures with osteoconductive forged unsintered hydroxyapatite and poly-L-lactide composite internal fixation devices. J Oral Maxillofac Surg 72: 1328-1338, 2014.

20. Culemann U, Holstein JH, Köhler D, Tzioupis CC, Pizanis A, Tosounidis G, Burkhardt M and Pohlemann T: Different stabilisation techniques for typical acetabular fractures in the elderly - a biomechanical assessment. Injury 41: 405-410, 2010.

This work is licensed under a Creative Commons Attribution-NonCommercial-NoDerivatives 4.0 International (CC BY-NC-ND 4.0) License. 